\title{
An operational dropping model towards efficient aerial firefighting
}

\author{
J. H. Amorim, C. Borrego \& A. I. Miranda \\ CESAM \& Department of Environment and Planning, \\ University of Aveiro, Portugal
}

\begin{abstract}
Forest fires have multifaceted impacts on societies. When integrated in an effective firefighting strategy, the rapid access of aerial resources to remote areas can play a crucial role. However, the efficiency of an aerial drop, due to the erratic atmospheric conditions and reduced visibility, depends essentially on the pilot's expertise since on-board systems for computer-assisted drops are unavailable. This work addresses the development of a numerical model for the real-time simulation of an aerial drop of firefighting products. The gas-phase is modelled through a vegetative canopy model coupled to a modified surface-layer model under diabatic conditions. For the particulate phase, the primary breakup of the retardant jet is obtained through the continuous stripping of droplets from the exposed surfaces by Rayleigh-Taylor and Kelvin-Helmholtz instabilities. The formed droplets are then subjected to distortion and secondary breakup due to aerodynamic forces mostly based on experimental correlations defined in terms of the Weber number. During the process, droplets are dispersed applying a Lagrangian approach. The code is linked to a GIS-based graphical interface, thus privileging the use by operational people. Computed ground pattern has been compared, with generally good results, with experimental data from a set of real scale dropping tests in which both conventional and constant flow-rate delivery systems were used. The model has potential to be used as a demonstrative application in firefighters training; as an operational tool during firefighting operations; or in the development of new products, as an alternative method to real scale dropping tests.
\end{abstract}

Keywords: aerial firefighting, operational numerical model, drop optimization. 


\section{Introduction}

Due to the increase on the frequency and severity of large wildland fires and wildland-urban interface fires, these have been identified by the World Health Organization as one of the threats to public health security in the $21^{\text {st }}$ century. Within this framework, the aerial drop of firefighting products can play, if integrated into an effective global strategy, an important role towards the protection of human lives and patrimony particularly in situations requiring a rapid intervention, as emerging fires, inaccessible mountainous areas, or highlyrisk areas. Since on-board systems for computer-assisted drops are not yet available, the efficiency of aerial means is, however, extremely dependent on the pilot skills in dealing with the fire characteristics, the complex atmospheric conditions and the reduced visibility. In this context, the development of numerical modelling tools can be of primary importance during training operations or real firefighting situations.

The design objective of an aerial delivery system is to guarantee an efficient ground coverage under adverse meteorological conditions, while reducing the environmental impacts (mainly on aquatic systems) associated to drift. However, prior to reaching the target, the behaviour of the liquid is determined by the balance of forces involved during breakup and dispersion of the fluid (see Figure 1). Initially, the relative velocity between the liquid and the gas phases will cause the deflection of the jet column. Simultaneously, the bulk product undergoes into a sequence of complex breakup mechanisms that transform it into ligaments and droplets with variable sizes that constitute the spray region with the typical cloud shape. This is further complicated by the fact that droplets are formed by a twostage breakup process, constituted by a primary and secondary breakup, resulting on a wide range of sizes. Moreover, droplets dynamics within the gaseous flow is governed by a number of factors, the importance of each is dependent on the droplet size as well as on the characteristics of the airflow, the latter intimately dependent on the roughness typology of the surface. The process ends with the gravitational settling of the product, through the penetration and coating of the canopy.

Few numerical models for the description of the dropping process in its entire extension have been developed, the most relevant by the US Forest Services

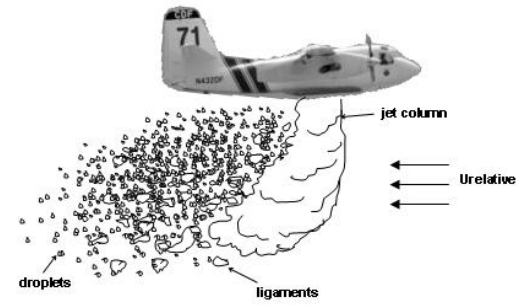

Figure 1: Schematic representation of the cascade of fluid structures originated by the breakup of a liquid jet during an aerial drop. 
(USDA-FS) and by research teams in Portugal and France under the EC projects ACRE and ERAS. The mechanisms that control the aerodynamic breakup of the product and the interaction of the formed droplets with the involving atmosphere, in particular under the influence of canopy-induced flows, are still unclear.

The objective of the current work is the development and validation of the operational Aerial Dropping Model ADM, intended for the simulation of the behaviour of firefighting liquids in the atmosphere, the time and space evolution of the cloud and the surface wetting pattern, with the ultimate goal of optimising the aerial dropping of firefighting products.

\section{Aerial dropping model development}

The model is divided into six modules, as represented in Figure 2, each one representing a stage on the aerial drop of the liquid. The input data are constituted by a series of parameters that describe the product characteristics, the flight, the meteorological conditions and the canopy characteristics. ADM generates a set of output data, from which the most relevant is the spatial distribution of liquid concentration at ground and/or canopy that allows the definition of the ground pattern contours.

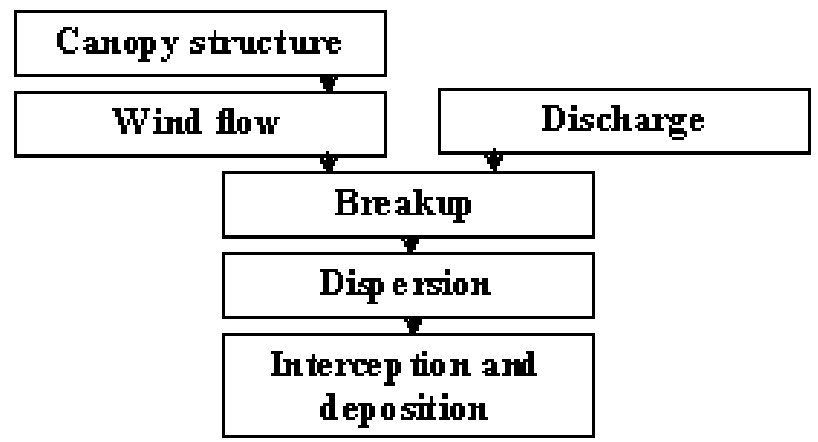

Figure 2: $\quad$ ADM model general fluxogram.

The different modules are hereafter briefly described. The tridimensional vegetative canopy-induced wind flow under diabatic conditions is simulated based on the proposal by Harman and Finnigan [1]. Vertical turbulent fluxes are calculated through a set of modified flux-profile relationships valid in the atmospheric roughness sublayer, which depart from the Monin-Obukhov Similarity Theory (MOST).

An approach for the definition of the canopy structure through the concept of Leaf Area Index (LAI) is implemented. This parameter, defined as one half the total leaf area per unit ground surface area, is an important structural characteristic of the forest ecosystem and one of the most important biophysical parameters on climatic, ecological and agronomical research studies. The user 
has the possibility to define the LAI value or to select it, depending on the tree species, from a list of default values. The vertical profile of LAI is calculated based on the modified Weibull cumulative distribution function developed by Teske and Thistle [2] from an extensive database of deciduous and coniferous trees.

In order to calculate the volume of product released per time, the three types of aerial delivery systems are available: the conventional, the constant flow, and the pressurized (MAFFS). The flow-rate values are user-defined or calculated based on the algorithm for estimating the liquid outflow from the tank developed by Swanson et al. [3].

The general theory behind the primary breakup of the jet derives from the concepts and numerical approaches introduced in the field of fuel spray modelling, especially from the model by Beale and Reitz [4]. Due to the specific characteristics of the firefighting liquid jet, in particular the extremely larger diameter, a competing mechanism between Rayleigh-Taylor and KelvinHelmholtz waves occurring in the liquid-gas interface was developed. The sizes of the child droplets resulting from primary breakup are computed with the jet stability theory, which establishes that the droplet formation resulting from interfacial instability is controlled by the particular wave which grows the most rapidly. The sizes of the droplets are thus related to the wavelengths of the most unstable waves. The secondary breakup of the formed droplets is then simulated by first identifying the breakup regime, bag or shear, through the dimensionless numbers Weber and Ohnesorge (Oh). According to this, experimental correlations for the spatiotemporal evolution of breakup are applied based on the works by Chou et al. [5] and Madabhushi [6].

The trajectories of the droplets during breakup are described by a Lagrangian dispersion module. A methodology for the evaluation of the effect of nonsphericity over the drag of the free-falling firefighting droplets is implemented. At this level, the effect of liquid viscosity on deformation can be important for Oh values above 0.1 (e.g. [7]). In these cases ADM applies the experimental correlation by Ortiz et al. [8]. When the liquid viscosity does not affect deformation $(\mathrm{Oh}<0.1)$, ADM incorporates a dynamical drag model that accounts for the effect of droplet shape transformation on drag coefficient. First, the model calculates the increase on droplet frontal diameter during the deformation period applying the experimental correlations by Hsiang and Faeth [7] and Madabhushi [6]. Then, the dynamical drag models from Morsi and Alexander [9] and Haider and Levenspiel [10] are used depending on the droplets' sphericity.

The canopy interception module applies the concept of film thickness, introduced on studies related to rainfall interception. The default values for water derive from extensive field measurements found in literature for different tree species. For retardant, the data from the laboratorial work conducted under the scope of the EC project ACRE [11] is used.

Finally, a GIS-based graphical User Interface (UI) is available, which is a user-friendly tool that allows the model to be used by firefighters for training purposes. The UI's development resulted from collaborative work under the EC project ERAS [12]. 


\section{Dropping model validation with real scale drop tests}

ADM model validation consisted on the comparison of the simulated ground concentrations against a set of real scale dropping tests data, in which different products and delivery systems were used, permitting the analysis of model performance within a wide range of conditions and the response of ground deposition to changes in retardant rheology. The drops were conducted at Marseille, France, in 2000 under the scope of the ACRE Project (see Figure 3) and at Marana desert, US, in 2005 by the USDA-FS.
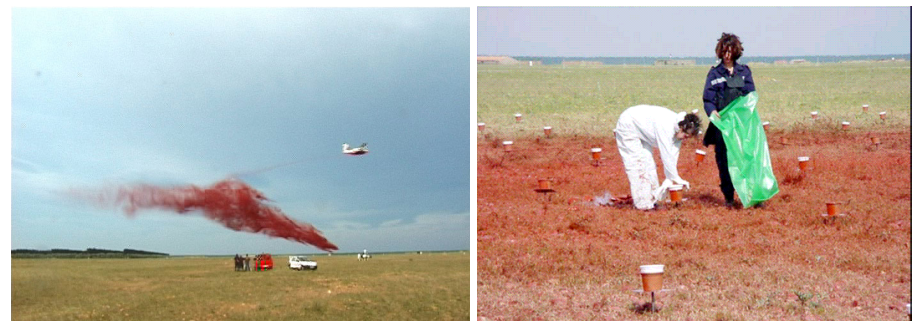

Figure 3: $\quad$ Retardant drop and cup collection at Marseille aerial drops [11].

The main characteristics of each dropping test are given in Table 1. In both drops a CDF-S2F aircraft was used, although with distinct tanks (higher volume in Marana) and delivery systems. A wide range of product viscosities was tested. Drop heights are considerably different, exceeding the $70 \mathrm{~m}$ in Marana. In both meteorological conditions were variable although most frequently with headwinds (opposite wind and aircraft directions). An important benefit from the Marana tests is that reliable data on instantaneous flow-rate was collected in flight from an on-board data acquisition system. In the simulation of Marseille drops the unavailability of flow-rate values required their calculation with the discharge module.

Measurements of product concentration at ground followed the "cup-andgrid" method, according to which a grid of cups is used and the weight of

Table 1: Dropping tests characterisation.

\begin{tabular}{|c|c|c|c|c|c|}
\hline Location & $\begin{array}{c}\text { Delivery } \\
\text { system }\end{array}$ & Retardant & $\begin{array}{c}\text { Viscosity } \\
(\mathrm{cP})^{*}\end{array}$ & $\begin{array}{c}\text { Drop } \\
\text { height } \\
(\mathrm{m})\end{array}$ & $\begin{array}{c}\text { Wind } \\
\text { velocity } \\
\left(\mathrm{m}_{\mathrm{s}} \mathrm{s}^{-1}\right)\end{array}$ \\
\hline Marseille & $\begin{array}{c}\text { Convent. } \\
\text { (salvo } \\
\text { drop) }\end{array}$ & $\begin{array}{c}\text { Fire-Trol } \\
931\end{array}$ & $432-1430$ & $34-45$ & $1-7$ \\
\hline Marana & $\begin{array}{c}\text { Constant } \\
\text { flow }\end{array}$ & $\begin{array}{c}\text { Phos- } \\
\text { Chek LV } \\
\text { MV and } \\
\text { HV }\end{array}$ & $152-1300$ & $38-78$ & $0.5-4$ \\
\hline
\end{tabular}

* The viscosity is expressed in the CGS unit system as centipoise: $1 \mathrm{cP}=10^{-3} \mathrm{~Pa} . \mathrm{s}$. 
retardant in each one is registered after total deposition (see Figure 3). Measured data are then interpolated in order to estimate the values between cups [13].

The validation procedure consisted on the intercomparison of the ground patterns shape, plus the statistical analysis of computed data in comparison to measurements, in terms of the length and area of each isoconcentration contour (or coverage level). Due to the distinct properties of the product, the analysis was performed using, in each case, the coverage levels typically applied in the US and in Europe, while facilitating the comparison with data from literature. Marana concentrations are thus given in gpc units (US gal.100 $\mathrm{ft}^{-2}=0.411 . \mathrm{m}^{-2}$ ).

Some examples of the simulated and measured ground patterns are shown in Figure 4 and Figure 5. Since the position of the aircraft at the instant of release is unknown, the locations of the modelled and measured patterns in the grid are not comparable. In general, ADM allows a good representation of the spatial distribution of product for the different coverage levels. The downrange and crossrange distribution, the formation of concentration 'islands' across the longitudinal axis of the pattern and the accumulation of product at the front in the conventional delivery system (Marseille) are generally well captured by the model. In some cases, computed results underestimate the accumulation at the pattern's front and the line lengths for the lower coverage levels.
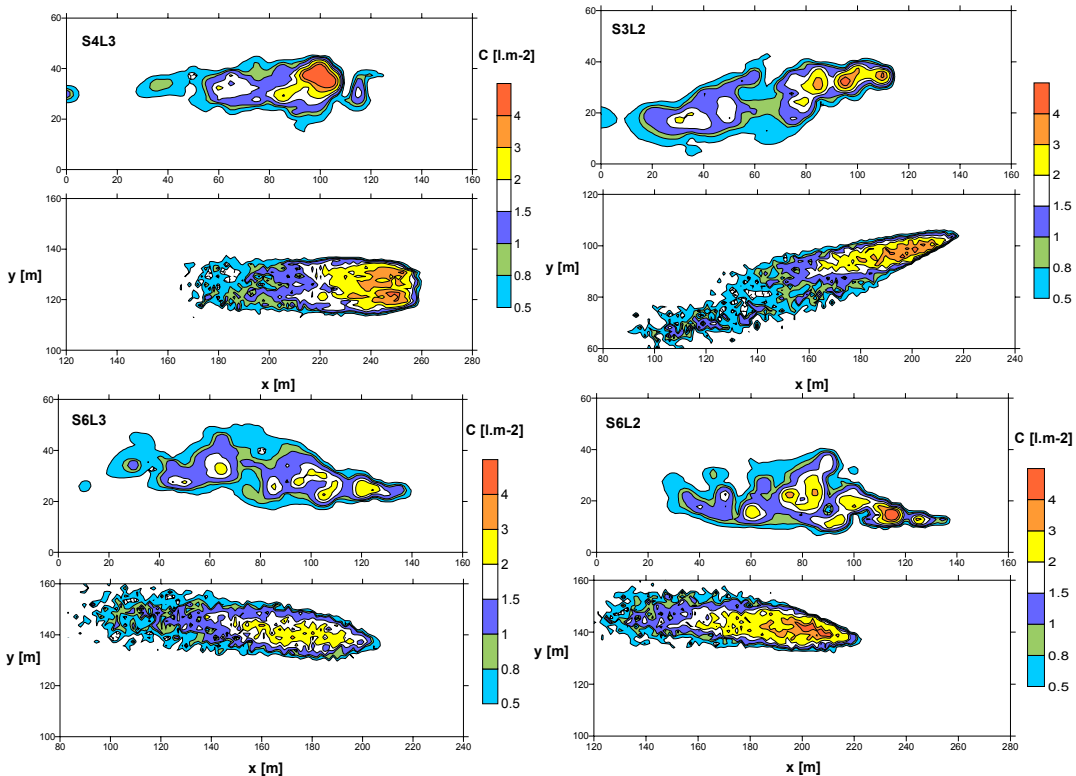

Figure 4: Comparison between the measured (up images) and simulated (down images) ground patterns of product concentration for a series of Marseille 2000 drop tests: $432 \mathrm{cP}$ (S4L3), $720 \mathrm{cP}$ (S3L2), $1060 \mathrm{cP}$ (S6L3) and $1430 \mathrm{cP}$ (S6L2). The colour scale indicates the minimum threshold concentration (in $1 . \mathrm{m}^{-2}$ ) of each coverage level. 
Due to the constant flow gating system the contours from the Marana tests are much more homogeneous than in Marseille. Results are shown in Figure 5.
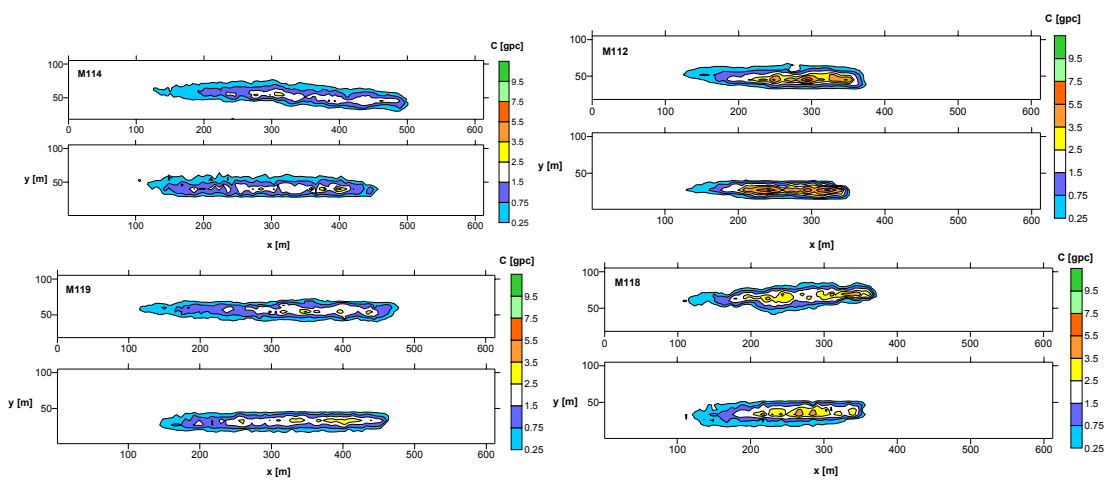

Figure 5: Comparison between the measured (up images) and simulated (down images) ground patterns of product concentration for a series of Marana 2005 drop tests: $152 \mathrm{cP}$ (M114), $214 \mathrm{cP}$ (M112), $1250 \mathrm{cP}$ (M119) and $1300 \mathrm{cP}$ (M118). The concentration values in the colour scale are given in gpc units.

In Figure 6, the validation metrics for the statistical intercomparison between the modelled and measured lengths and areas of each coverage level are presented. The averaged normalised mean squared error (NMSE) for the computed contours lengths is 0.015 ; while the NMSE for the simulated areas occupied by each level increases to 0.046 . The averaged Pearson correlation coefficients (not shown) are 0.989 in both cases. The accuracy of the simulations shows no strong relation with the corresponding viscosity, although better results are obtained in the range from 700 to $1100 \mathrm{cP}$. Except for one case, there is a decreasing tendency of the NMSE relating the simulated contour areas with the increasing viscosity.

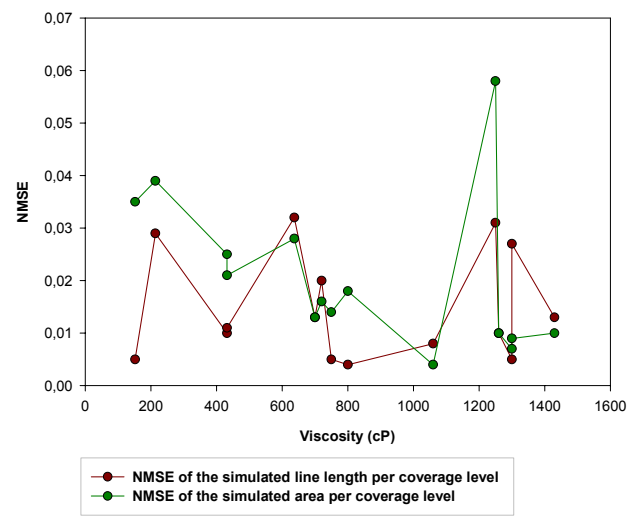

Figure 6: NMSE of the simulated length and area of each ground coverage level, for the entire range of viscosities tested. 
As an example, Figure 7 shows a comparison between modelled and measured lengths for each coverage level. The modelling goal was to maintain in each level the computed values within $10 \%$ error (shown by the vertical error bars) from the measured lengths. In the example only level 6 (corresponds to concentrations in the range $3-41 . \mathrm{m}^{-2}$ ) does not fulfil this requirement.

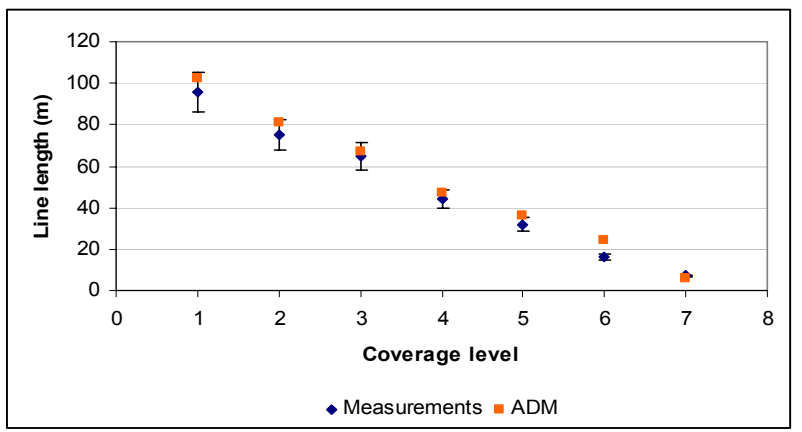

Figure 7: Comparison between modelled and measured line lengths per coverage level for the S6L1 $(1260 \mathrm{cP})$ Marseille dropping test. The minimum threshold concentrations for the different levels are the following: 1: $0.51 . \mathrm{m}^{-2} ; 2: 0.81 . \mathrm{m}^{-2} ; 3: 11 . \mathrm{m}^{-2} ; 4: 1.51 . \mathrm{m}^{-2}$; 5: $21 . \mathrm{m}^{-2} ; 6: 31 . \mathrm{m}^{-2} ; 7: 41 . \mathrm{m}^{-2}$.

In general, the results can be considered satisfactory given the wide range of input conditions and the difficulty in simulating the complex dynamical behaviour of retardants in the atmosphere while maintaining the operational characteristics of the model.

\section{Conclusions}

The main objective of the present work was the development and evaluation of a model for the numerical simulation of the aerial dropping of firefighting products, covering the different stages and mechanisms involved: canopy-induced wind flow; outflow of the liquid from the aircraft tank; jet column bending and fracture; primary breakup of the jet surface and column; shape distortion and secondary breakup of the formed droplets; gravitational settling through transport and dispersion mechanisms; and canopy interception of the spray cloud.

The model performance was investigated against real scale measured data. A wide range of input conditions, as delivery system type, flight parameters, meteorological conditions, and products characteristics were tested. The computed ground pattern shapes show the features observed in measured data. The statistical validation of the results in terms of the computed length and area for a number of concentration ranges (coverage levels) indicated a generally good model performance, in particular given the wide range of input conditions assessed. Line lengths per coverage level are within a $10 \%$ error in general, with averaged NMSE of 0.015 and Pearson correlation coefficient of 0.989 . The 
accuracy on the simulated areas per level decreases to an averaged NMSE of 0.046 , although the good correlation maintains.

Results have shown that, in some situations, the longitudinal dispersion of droplets is underpredicted for lower liquid viscosities, resulting in shorter patterns. Possible reasons for this could be related with some features which are not encompassed in the modelling, namely the effect of the wake induced by the flow around the aircraft and the jet column over the motion of the smaller droplets, in particular those formed by Kelvin-Helmholtz instabilities originated in the surface of the jet and spray cloud.

The investigation of the spatiotemporal evolution of primary breakup of the jet, with significant impact on final result, should be stimulated. Also the knowledge of the response of viscosity to shear stress due to the non-Newtonian characteristics of retardants would increase the accuracy of modelling results, particularly for those fluids showing elastic properties (as Phos-Chek). Only a systematic and detailed laboratorial analysis could provide these data.

The model is primarily vacationed for 'indirect attacks' situations, in which the drop is made at some distance from the fire front, thus allowing the effect of temperature on wind flow patterns to be neglected. A wider application field could be obtained if ADM was integrated with a fire progression model.

Due to its characteristics and capabilities, namely the fast-running code (about 1 minute CPU time) and the friendly-access graphical UI, this operational tool can potentially be used in the formation, training and demonstration activities with pilots, aerial resources coordinators, civil protection personnel or general firefighters, or in the testing of the effectiveness of firefighting chemicals, complementing the data obtained from real scale drop tests and laboratorial experiments. The user control over the input parameters allows the effect on ground pattern to be assessed for a wide range of release scenarios, avoiding the natural variability and irreproducibility of field conditions, and a better understanding of the multiple interrelated phenomena involved.

\section{Acknowledgements}

The authors are especially grateful to Eng. Ryan Becker and Eng. Greg Lovellette from the USDA-FS and to Dr. Ian Harman from the CSIRO Marine and Atmospheric Research. The authors would like to acknowledge also the financial support of the $3^{\text {rd }}$ European Framework Program through the Projects INTERFACE (POCI/AMB/60660/2004), ERAS (EVG1-2001-00019) and EUFIRELAB (EVR1-CT-2002-40028), and the Portuguese "Ministério da Ciência e do Ensino Superior", through the "Fundação para a Ciência e Tecnologia", for the PhD grant of J.H. Amorim.

\section{References}

[1] Harman I. and Finnigan J., 2007. A simple unified theory for flow in the canopy and roughness sublayer. Boundary-Layer Meteorology 123(2), 339-363. 
[2] Teske M.E. and Thistle H.W., 2004. A library of forest canopy structure for use in interception modelling. Forest Ecology and Management 198(1-3), 341-350.

[3] Swanson D.H., Luedecke A.D. and Helvig T.N., 1978. Experimental tank and gating system (ETAGS). Final report, contract 26-3425, 1 September 1978, Honeywell Inc., Government and Aeronautical Products Division. Hopkins, Minnesota, USA. 62 p.

[4] Beale J.C. and Reitz R.D., 1999. Modeling spray atomization with the Kelvin-Helmholtz/Rayleigh-Taylor hybrid model. Atomization and Sprays 9(6), 623-650.

[5] Chou W.H., Hsiang L-P. and Faeth G.M., 1997. Temporal properties of secondary drop breakup in the shear breakup regime. Int. J. Multiphase Flow 23, 651-669.

[6] Madabhushi R.K., 2003. A model for numerical simulation of breakup of a liquid jet in crossflow. Atomization and Sprays 13. 413-424.

[7] Hsiang L.-P. and Faeth G.M., 1992. Near-limit drop deformation and secondary breakup. International Journal of Multiphase Flow 18(5), 635652 .

[8] Ortiz C., Joseph D.D. and Beavers G.S., 2004. Acceleration of a liquid drop suddenly exposed to a high-speed airstream. International Journal of Multiphase Flow 30, 217-224.

[9] Morsi S.A. and Alexander A.J., 1972. An investigation of particle trajectories in two-phase flow systems. J. Fluid Mech. 55(2), 193-208.

[10] Haider A. and Levenspiel O., 1989. Drag coefficient and terminal velocity of spherical and nonspherical particles. Powder Technology 58, 63-70.

[11] Giroud F., Picard C., Arvieu P. and Oegema P., 2002. An optimum use of retardant during the aerial fire fighting. In: IV International Conference on Forest Fire Research, Luso, Portugal, 18-23 November 2002 - Forest Fire Research \& Wildland Fire Safety, Viegas, D.X. (Ed.), Millpress, Rotterdam. Proceedings in CD-Rom.

[12] Amorim J.H., Miranda A.I., Borrego C. and Varela V., 2006. Recent developments on retardant aerial drop modelling for operational purposes. In: V International Conference on Forest Fire Research, Figueira da Foz, Portugal, 27-30 November 2006 - Forest Fire Research \& Wildland Fire Safety, Viegas, D.X. (Ed.), Millpress, Rotterdam. Proceedings in CD-Rom.

[13] Lovellette G., 2004. How to conduct drop tests of aerial retardant delivery systems. Tech. Rep. 0457-2813-MTDC. Missoula, MT: U.S. Department of Agriculture, Forest Service, Missoula Technology and Development Center. $18 \mathrm{p}$. 